\title{
A hypertension control quality improvement pilot program: Experiences and blood pressure outcomes from physician practices
}

\author{
Tamkeen Khan*1, Jianing Yang ${ }^{1}$, Laken Barkowski ${ }^{1}$, Beth Tapper ${ }^{1}$, Lisa Lubomski ${ }^{2}$, Donna Daniel ${ }^{3}$, Gregory Wozniak ${ }^{1}$ \\ ${ }^{1}$ American Medical Association, Chicago, Illinois, United States \\ ${ }^{2}$ Johns Hopkins University School of Medicine, Baltimore, Maryland, United States \\ ${ }^{3}$ IBM Watson Health, Cambridge, Massachusetts, United States
}

Received: February 22, 2018

DOI: $10.5430 /$ ijh.v4n1p42
Accepted: March 22, 2018

Online Published: March 28, 2018

\begin{abstract}
Improving Health Outcomes: Blood Pressure (IHO: BP) was a Quality Improvement (QI) pilot program developed by the American Medical Association (AMA) and Johns Hopkins Medicine aimed at helping physicians and their care teams better manage patients with uncontrolled hypertension. The pilot study was conducted at 10 ambulatory practice sites between October 2013 and November 2014 in which the initiative was to devise a framework and intervention strategies for improving hypertension control. The program included evidence-based tools and materials to support the Measure Accurately, Act Rapidly, and Partner with Patients (M.A.P.) framework, interactive components with coaching support and peer-to-peer learning opportunities, and a measurement system supported by health technology, all intended to aid blood pressure management. This paper captures the learnings from the engagement, experiences, and satisfaction of care teams from the $I H O: B P$ pilot that were used to adjust, reassess, and refine components of the QI program. Overall, participation in the IHO: $B P$ pilot was associated with an increase in BP control rates from a mean of $69 \%$ to $75 \%(p<.05)$ for 3 of the 10 practices. Mean systolic and diastolic blood pressure was reduced in 8 of 10 practices by a mean of $12.5 \mathrm{mmHg} / 6.5 \mathrm{mmHg}(p<.05)$. Furthermore, evaluation of participant experiences indicated that $75 \%$ of the respondents were satisfied or very satisfied with the initiative. The results from this study include components of the pilot that participants indicated were most helpful and were used to generate useful information for hypertension QI efforts that were later scaled and spread to subsequent initiatives.
\end{abstract}

Key Words: Hypertension, Quality improvement, Program implementation, Ambulatory QI

\section{INTRODUCTION}

Hypertension control is a crucial component for preventing cardiovascular disease, a factor in more than 400,000 premature deaths in 2014. ${ }^{[1]}$ In the United States, nearly 86 million adults have hypertension and approximately 16 million of these individuals do not have their blood pressure under control (BP, $\mathrm{mmHg}$ ) of $\geq 140$ systolic and/or $\geq 90$ diastolic despite having a usual source of health care, being aware of their diagnosis, and receiving treatment for their condition. ${ }^{[2]}$ Reasons for the lack of hypertension control may include, but are not limited to, blood pressure measurement errors in clinical settings, insufficient treatment or therapeutic inertia (TI) which is the failure to initiate or intensify pharmacotherapy when blood pressure measurements are uncontrolled, or suboptimal adherence to the prescribed hypertension therapy. Achieving hypertension control of $70 \%$ or greater among

*Correspondence: Tamkeen Khan; Email: Tamkeen.Khan@ama-assn.org; Address: American Medical Association, Chicago, IL, United States. 
patients currently under clinical care could prevent approximately 50,000 deaths per year. ${ }^{[3,4]}$

To address these gaps in hypertension control the American Medical Association (AMA) partnered with the Johns Hopkins Medicine (Johns Hopkins) to develop the Improving Health Outcomes: Blood Pressure (IHO: BP) quality improvement (QI) program focused on helping physicians and their care teams better manage patients with uncontrolled hypertension. The IHO: $B P$ pilot aimed to implement and test an ambulatory QI model developed using evidence based research and evaluation strategies. QI collaborations have been shown to improve blood pressure control rates ${ }^{[5,6]}$ and a few studies have determined which components are most helpful to chronic care management. ${ }^{[7-10]}$

The purpose of this study is to expand the literature on hypertension control improvement collaborations and present the learnings from the implementation of the IHO: BP pilot program in order to assess and refine components of the program for subsequent initiatives. The results from the pilot were not expected to create a dynamic shift or significant improvements in outcomes or BP control rates. The primary intention of the study was to provide information and generate participant feedback on the feasibility and usefulness of the components of the pilot interventions that could be incorporated into the next generation of hypertension QI programs that would be subsequently scaled and spread. The results may also be useful to others implementing QI pilots. This paper begins with an overview of the $I H O: B P$ prototype pilot. It then describes the study procedures used to obtain clinical and intermediate outcomes from the pilot, as well as capture experiences of participating care teams. Finally, the clinical outcomes and participant satisfaction experiences are discussed along with their implications for future QI projects.

\section{IHO: BP QI PROGRAM}

\subsection{Overview}

The IHO: $B P$ pilot involved engaging physicians and care teams in managing hypertension control among their patients and consisted of three main components including (1) evidence-based framework of tools and materials to aid in blood pressure management, (2) interactive components with coaching support and peer-to-peer learning opportunities, and (3) a measurement system supported by health technology to demonstrate the importance of accurate and credible data in achieving progress and assisting in patient outreach. The pilot was conducted between October 2013 and November 2014 in 10 ambulatory practices and health centers, 5 in the Chicago Illinois metropolitan area and 5 in Maryland. These practices and health centers differed in size, practice type, and patient characteristics. ${ }^{[11]}$

Published by Sciedu Press

\subsection{Evidence-based tools and materials}

The IHO: BP program provided a standard set of tools and materials to all participating sites. An evidence-based checklist, referred to as the Measure Accurately, Act Rapidly, and Partner with Patients (M.A.P.) checklist, identifies three clinically-intuitive domains for managing patients with uncontrolled hypertension: M.A.P., Families and Communities. ${ }^{[12,13]}$ The Measure Accurately checklist aims to help clinical care teams ensure the blood pressure measurements they obtain are most representative of a patient's true blood pressure. The Act Rapidly checklist aims to help clinical care teams decrease TI and promptly begin treatment of hypertension using an evidence-based treatment algorithm once a diagnosis is made. This is done by helping clinical care teams measure the extent to which TI occurs in their practice and discover likely causes in efforts to identify opportunities for improvement. The Partner with Patients, Families and Communities checklist aims to empower and support patients in self-management of their disease by using strategies for engaging patients, helping them to accurately self-measure their blood pressure and providing patients and families with referrals to resources that support medication adherence and healthy lifestyle interventions. Additional tools and materials provided to the practice sites consisted of posters containing a visual and written depiction of proper patient positioning during blood pressure measurements in order to obtain a reading that is representative of a patient's true blood pressure, a Practice Context Assessment (PCA) ${ }^{[11]}$ survey administered at the beginning and end of the pilot to measure changes in the culture of the practice to better understand elements that might affect hypertension control improvement efforts, and a Gauging Progress Survey (GPS) that allowed sites to assess QI implementation efforts and identify areas for further improvement. [14]

\subsection{Interactive components}

The IHO: BP pilot consisted of two interactive components. Physicians and members of their care teams from participating sites were brought together for eight educational webinars around the three M.A.P. focus areas. These webinars allowed participants to discuss the successes and barriers encountered in the implementation of the $I H O: B P$ program and peer-to-peer collaboration to identify solutions to those barriers. Practice sites were also brought together for quarterly in-person learning events which were designed to create networking opportunities for the clinical care teams, strengthen relationships with the $I H O: B P$ project team, demonstrate effective use of the tools and resources, share strategies for improving blood pressure control, and identify solutions for program improvement. 


\subsection{Health technology measurement system}

The IHO: BP program provided the 10 participating practices with automated BP machines as part of an accurate measurement intervention intended to provide exact BP measurements rather than less precise, rounded measurements based on a manual aneroid sphygmomanometer BP measurement. Patient clinical data was extracted from each sites' electronic health record system by Forward Health Group (FHG) for performance tracking. The sites were also given access to FHG's PopulationManager ${ }^{\circledR}$ which is a customized online population health data management platform that allowed sites to view their blood pressure control rates, identify patients with uncontrolled blood pressure and perform patient outreach to have uncontrolled patients come in for a checkup. The data platform demonstrated the importance of using credible data to drive hypertension control improvement efforts. ${ }^{[2,15]}$

\section{METHODS}

The primary outcomes for the study were pre- and postintervention BP control rates at each site; average systolic blood pressure/diastolic blood pressure (SBP/DBP) among patients with uncontrolled blood pressure; TI defined as the percentage of encounters with documented uncontrolled BP among patients with a hypertension diagnosis and no change in medication; terminal digit preference (defined as the percentage of BP readings ending in zero); and overall participant satisfaction with the IHO: BP program components. The IHO: $B P$ team also utilized the data extracted by FHG to evaluate these outcomes. The study population included patients between the ages of 18-85 years with a diagnosis of hypertension from the 10 practice sites. Eligible patients had at least one office visit with a recorded BP in their electronic health record in 2014. Patients who met the exclusion criteria of the National Quality Forum Measure 18 for hypertension control were excluded. Two-tailed $t$-tests were performed to assess differences between pre- and post-intervention in BP control rates and for changes in the mean SBP and DBP for uncontrolled patients. Joinpoint regression analysis of trends in TI were evaluated for participants and a comparison group (patients from nonparticipating physicians at the 10 sites) using Joinpoint Regression Program, Version 4.2.0.2 (Statistical Research and Applications Branch, National Cancer Institute). This method was applied to identify significant changes in trends, and a maximum of three joinpoints was allowed for each estimation.

Participant satisfaction data were collected from an online Site Experience Survey (SES) at the end of the pilot to specifically quantify usefulness of the various $I H O: B P$ components to identify areas that were most helpful, and determine elements that could be improved. The survey was sent to actively engaged participants including physicians, nurses, and physician assistants who had taken the PCA survey and attended at least 1 interactive webinar or in-person learning event. Satisfaction ratings ( 1 to 5 on a Likert scale where $1=$ very dissatisfied and $5=$ very satisfied) with were collapsed into three categories: satisfied (satisfied or very satisfied), neutral and dissatisfied (dissatisfied or very dissatisfied). For items gauging the helpfulness of other components, we obtained the mean results on a scale of 1-3 (score of $1=$ not helpful, score of 2 = helpful, score of 3 = very helpful). Utilization of the M.A.P framework tools was measured by questions acknowledging the use of the tools (yes or no). For items measuring level of agreement, mean results were obtained on a scale of 1-4 (score of $1=$ strongly disagree, score of $2=$ disagree, score of $3=$ agree and score of $4=$ strongly agree). With the exception of the joinpoint regressions, we used SAS version 9.4 (SAS Institute, Cary, NC) and STATA 13 (StataCorp LP, College Station, TX) software for all analyses. This study was deemed exempt from Institutional Review Board approval by the Office for the Protection of Research Subjects at the University of Illinois at Chicago and the Office of Human Subjects Research at John Hopkins Medicine.

\section{RESULTS}

\subsection{BP control rates and mean SBP/DBP}

The results shown in Figure $1(n=26,235$ patients in 2013 and $n=28,847$ patients in 2014) suggest that participation in the IHO: BP program was associated with increased BP control rates for 3 of the 10 practices, from an average of $69 \%$ to $75 \%(p<.05)$. This may reflect reductions in terminal digit preference from the use of automated BP machines. Four practices had statistically significantly lower control rates, while 8 of the 10 practices saw the mean SBP/DBP reduced by $12.5 \mathrm{mmHg} / 6.6 \mathrm{mmHg}(p<.05)$.

The annual percent changes (APCs) are reported for the participants and non-participants using a log-linear model are shown in Figures 2 and 3. Trend in TI for the participant group showed a decrease during 2014 (APC -0.0043\%, $p$ $=.025)$; TI displayed a negative trend that was not statistically significant, in 2014 for the comparison group (APC = $-0.0051 \%, p=.068)$.

For patients whose blood pressure was uncontrolled in the TI measure, analyses of the BP control rate within 30 days of medication change and the mean number of days between visits is in Table 1 . The BP control rate for the participant group was higher than for the comparison group (0.50 vs. $0.43, p=.012)$; days between visits was higher for the participant than the comparison group (17.9 vs. $15.8, p<.0001)$. 


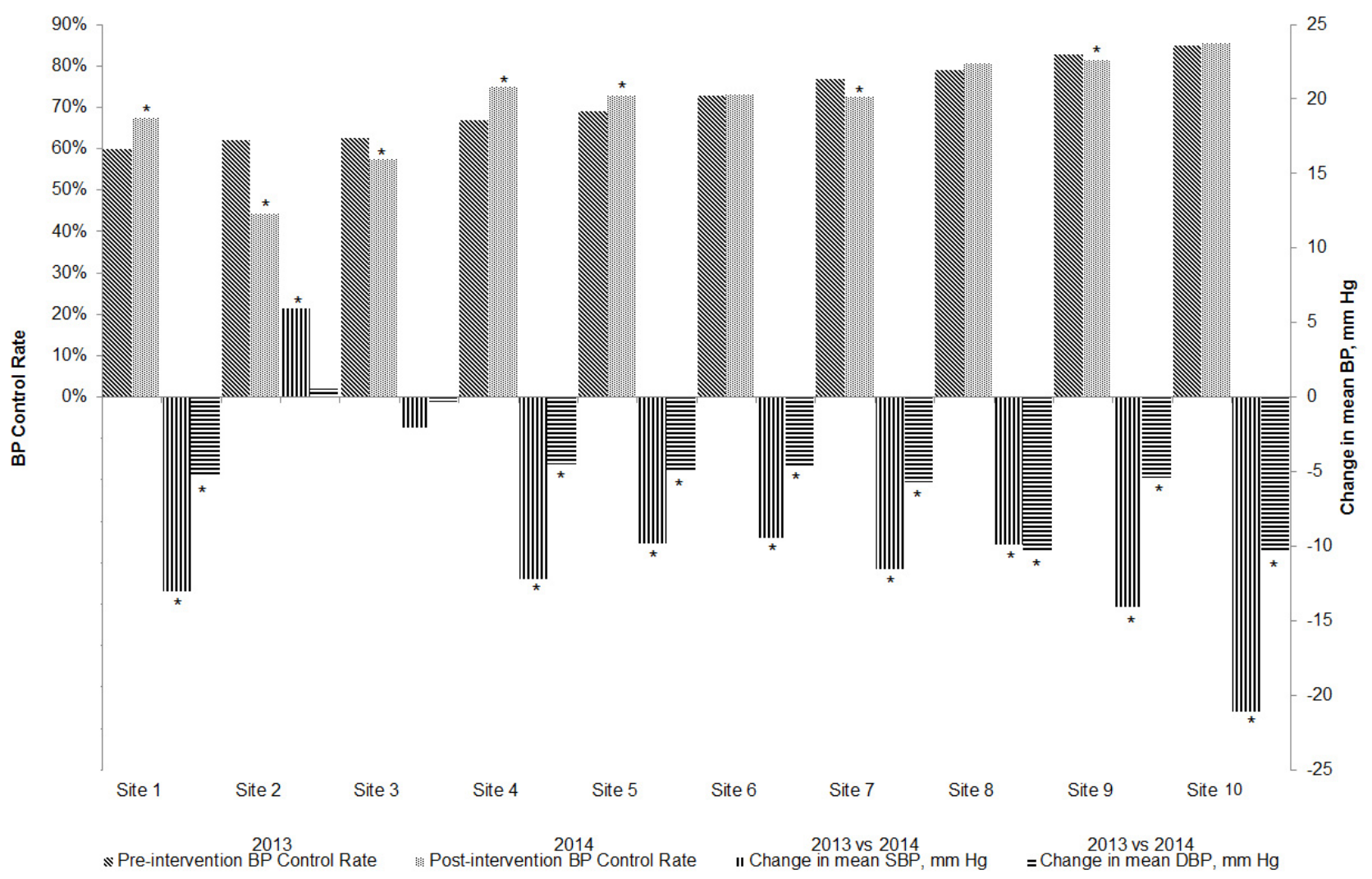

Figure 1. Site level changes in BP control rates and mean SBP/DBP for patients with uncontrolled BP pre-intervention (2013) vs. post-intervention (2014)

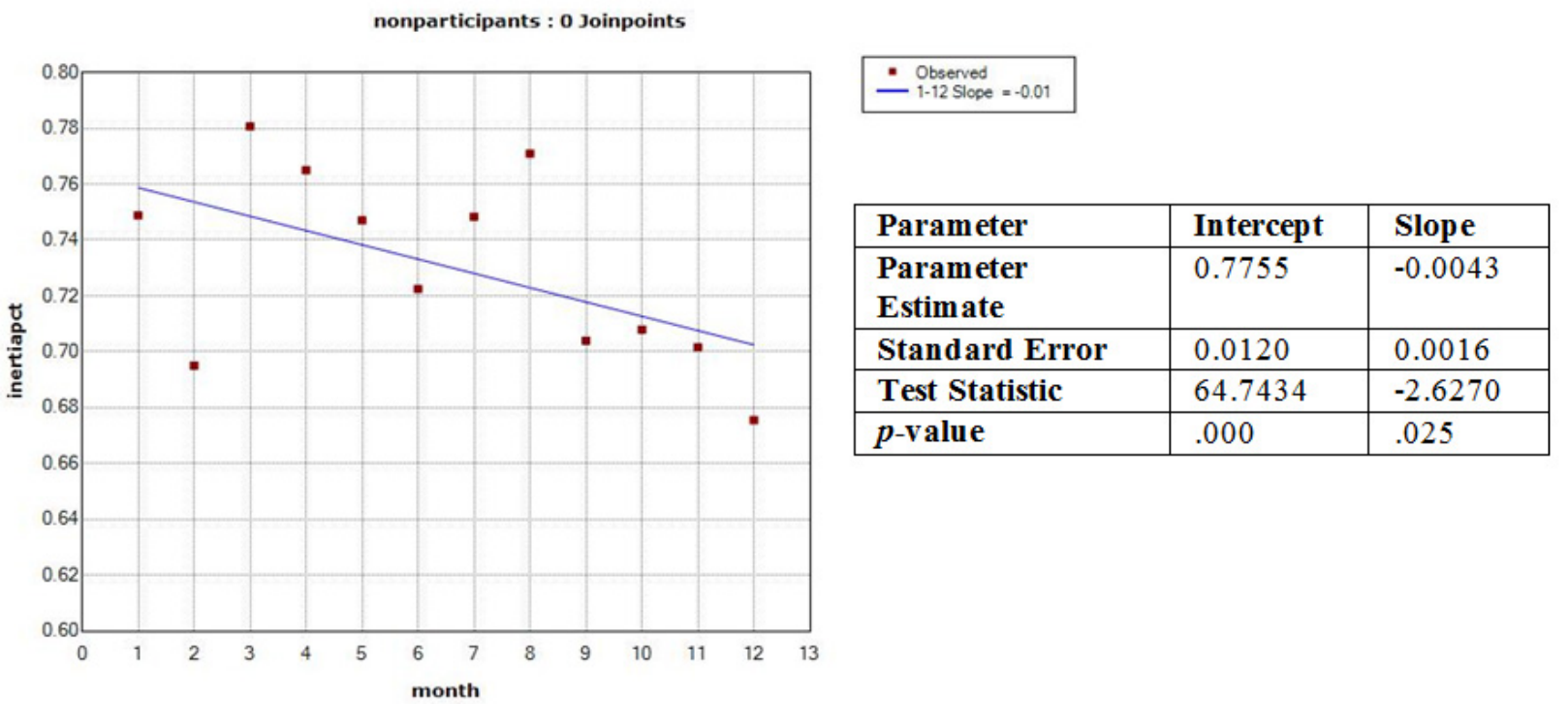

Figure 2. Trends in therapeutic inertia participants

Table 1. Differences in BP control rate and days between visits for uncontrolled patients with medication change

\begin{tabular}{lllll}
\hline Variable & Participant Group & Non-Participant Group & t-value & $\boldsymbol{p}$-value \\
\hline BP Control Rate & 0.50 & 0.43 & -2.51 & .012 \\
Days Between Visits & 17.9 & 15.8 & -4.60 & $<.0001$ \\
\hline
\end{tabular}



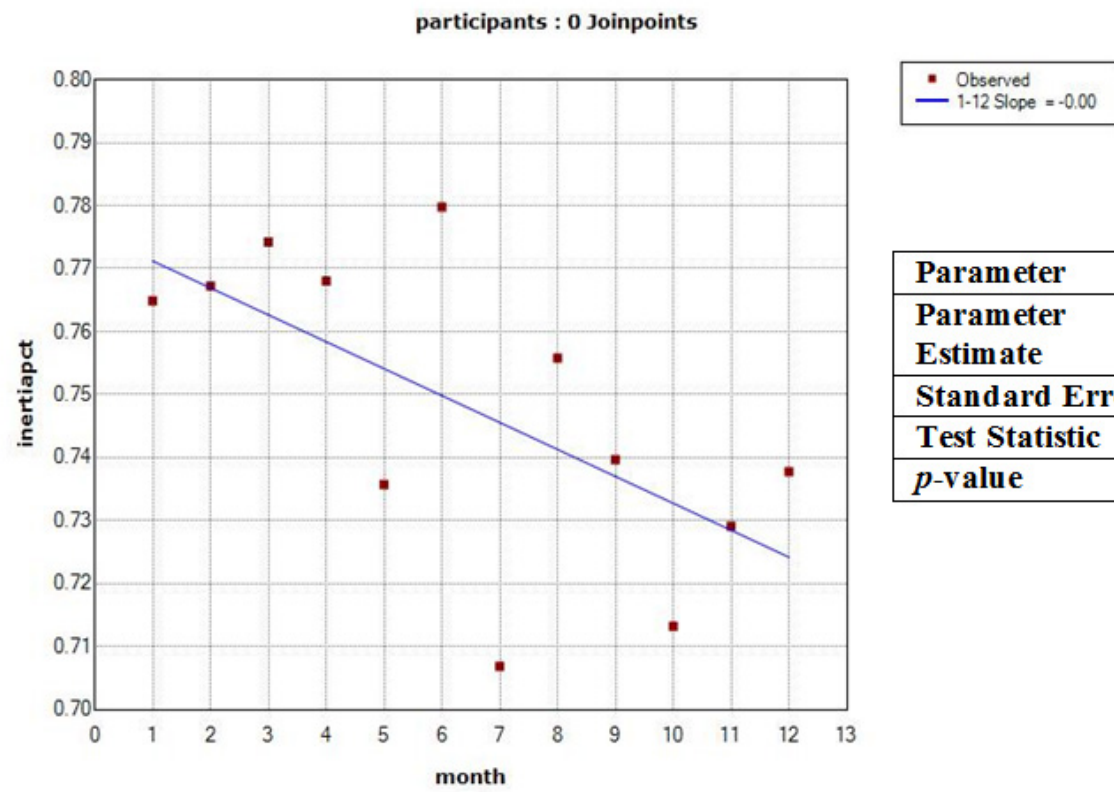

\begin{tabular}{|l|l|l|}
\hline Parameter & Intercept & Slope \\
\hline $\begin{array}{l}\text { Parameter } \\
\text { Estimate }\end{array}$ & 0.7639 & -0.0051 \\
\hline Standard Error & 0.0184 & 0.0025 \\
\hline Test Statistic & 41.5656 & -2.0495 \\
\hline$p$-value & .000 & .0676 \\
\hline
\end{tabular}

Figure 3. Trends in therapeutic inertia non-participants

\subsection{Participant satisfaction}

The SES was collected from 33 of the 47 participants to whom it was distributed yielding a response rate of $70.2 \%$. The sample included one or more respondents from each of the ten practice sites. Initially, 114 participants were eligible for the SES based on completion of the baseline PCA survey, however, 68 individuals (57.0\%) were excluded from this sample because they did not demonstrate active engagement in the program as defined by participating in an interactive component and 2 individuals (1.8\%) were excluded because they resigned from the practice prior to the conclusion of the program. The SES survey responses indicated that $75 \%$ of the respondents were satisfied or very satisfied with the initiative ( (mean score of 2.71 [SD $=0.52]$ ) on a scale of $1-3$ where $1=$ dissatisfied, and $3=$ very satisfied).

Table 2 provides the ratings for helpfulness of the various components of the IHO: BP program. The learning events were reported to be most helpful with an average value of $2.57(\mathrm{SD}=0.50)$ (on a scale of $1-3$ where $1=$ not helpful and $3=$ very helpful) and the webinars were the least helpful with an average value of $2.10(\mathrm{SD}=0.40)$.

Regarding the level of agreement for the core benefits of the IHO: $B P$ program on a scale of $1-3$, the overall results indicate that "improvements in delivery of primary care", and "changes in overall culture", ranked highest with mean scores of $3.48(\mathrm{SD}=0.71)$ and $3.47(\mathrm{SD}=0.75)$, respectively (results not shown). The level of agreement that the initiative "helped improve hypertension care for patients", "made administrative operations more efficient", and "helped understand the importance of blood pressure control" ranged from average scores of $3.39(\mathrm{SD}=0.61)$ to $3.34(\mathrm{SD}=0.69)$. On the lowest end of the scale was agreement with "aid in reducing health disparities" with a score of $3.09(\mathrm{SD}=0.84)$. Nonetheless, all of the mean scores indicate the IHO: BP program helped in several areas.

Table 2. Average scores for helpfulness of $I H O: B P$ program components (scores 1 to 3 , where $1=$ not helpful and $3=$ very helpful)

\begin{tabular}{ll}
\hline Program Component & Mean (SD) \\
\hline Learning Events & $2.567(0.504)$ \\
Posters & $2.484(0.626)$ \\
PopulationManager $^{\circledR}$ & $2.400(0.498)$ \\
M.A.P. Framework Tools & $2.379(0.561)$ \\
Gauging Progress Survey & $2.258(0.514)$ \\
Practice Context Assessment & $2.129(0.562)$ \\
Webinars & $2.097(0.396)$ \\
\hline
\end{tabular}

\section{DisCUSSION}

The IHO: BP pilot program was a multi-faceted QI approach comprised of several effective methodologies ${ }^{[9,10]}$ including evidence-based checklists to aid in implementation efforts, ${ }^{[16-18]}$ ongoing practice facilitation, ${ }^{[19,20]}$ opportunities for peer-to-peer learning, and the use of a health technology platform. ${ }^{[21,22]}$ The study applied multi-level observational data to evaluate the impact of the program on blood pressure control rates and other outcome measures. The comparison of pre- and post-intervention measurements indicated program participation resulted in marginal improvements 
in $\mathrm{BP}$ control rates, which is partially due to reductions in terminal digit preferences (data not shown) from the use of automated BP machines. Patients with poorly controlled hypertension showed significant reductions in mean SBP/DBP among the majority of participating sites. Program participation was also associated with a significant reduction in TI, an intermediate outcome measure associated with program interventions aimed at acting rapidly to manage uncontrolled BP.

\subsection{Learnings}

Collective feedback obtained from this pilot was used assess and modify various components of the hypertension QI pilot program so they could be used in subsequent hypertension control projects. As a result of this feedback, the tools and resources were refined as follows:

(1) Participants indicated that the in-person learning events were the most helpful component of the program, providing opportunities to share successes, exchange knowledge and strategies for improvement, and network with other peers. However, they also reported the virtual didactic webinars were the least helpful component on the program since they interrupted patient flow for medical practitioners and did not gain much value from the instruction of the informational content. Using this feedback, "Share Your Experience" webinars were created in the subsequent hypertension QI initiatives which combined the networking aspect of the in-person learning events with the virtual aspect of the webinars. In efforts to increase the benefits from these events, participants in the latter hypertension QI programs were provided early access to short pre-recorded educational podcasts with an accompanying one-page informational sheet, both of which can be reviewed at the participants convenience. ${ }^{[23-25]}$ Furthermore, the webinars were transformed into discussions between the various participants in which they shared the successes and barriers they encountered during implementation of the QI components. This new format increased flexibility and peer-to-peer learning opportunities for participants in the spread of subsequent hypertension initiatives.

(2) The second most helpful piece of the hypertension improvement program was the blood pressure measurement posters sent for display at the practice sites. As a result of this feedback, these were modified to show the most critical steps for measuring blood pressure accurately, the potential impact to measurement, and evidence-based recommendations for correct positioning. The posters have been shared with practices and health centers nationwide, and in an effort to easily distribute them on a large scale they are also available on the AMA and other collaborator websites. ${ }^{[26,27]}$ Some of these materials were converted into formats that would be available for display on electronic mobile applications.

(3) The M.A.P. framework tools were the core of the $I H O$ : $B P$ pilot. Although the overall helpfulness of the M.A.P. framework tools was 2.38 ( $\mathrm{SD}=0.56), 94 \%$ of the survey respondents reported utilizing the Measure Accurately tools, $84 \%$ reported using the Act Rapidly and $71 \%$ reported using the Partner with Patients tools. Participants reported that tools were useful; however the curriculum was dense and required a heavy time commitment. Based on these comments, the M.A.P. tools were refined into fast fact sheets which were intended to make it easier for practices to quickly incorporate the content. Furthermore, these tools have also been made readily available online for wider and easier access. ${ }^{[26]}$

(4) Finally, the survey components of the hypertension initiative were ranked on the low end for being helpful for hypertension QI efforts. The PCA and GPS were intended to measure changes in practice culture as well as assess the level of implementation of the IHO: $B P$ program, however they were quite lengthy. These items were refined in the spread of the QI materials and were replaced with more simplified baseline assessments such as the PCA v2.0 which was $45 \%$ shorter than the original version. ${ }^{[11]}$

\subsection{Limitations}

Although there have been some strong findings from the $I H O$ : $B P$ pilot in terms of care team engagement, experience and satisfaction, we do recognize several limitations. This study was a pilot test of a QI initiative and included a small number of primary care practices or health centers. Nevertheless, there was variation in the characteristics of each practice so it is likely that the experience of these teams is generalizable. Still, large-scale implementation of the IHO: $B P$ program will likely require more attention to local adaptation of implementation methods.

Additionally, as the program progressed, site implementation of evidence-based tools and checklists decreased. During the pilot, participants were first educated on $\underline{\text { Measure Accu- }}$ rately, then Act Rapidly and finally Partner with Patients. To address this issue in future iterations of the QI initiative, the revised IHO: $B P$ program incorporates more flexibility in the length of implementation; one version of the program is nine months and another version is six months. Future iterations 
of the IHO: $B P$ program may also allow participants to implement tools and interventions based on need, and forego areas in which the site is already performing well.

\section{Conclusion}

The IHO: BP program was a ground breaking effort in developing a foundation to evaluate the impact of a robust hypertension QI program on blood pressure control rates and other outcomes. Although further application and development of this initial work is still needed to better improve the management of patients with uncontrolled hypertension, participants from this pilot study provided critical feedback around the feasibility and usefulness of components of the QI framework that led to refinements and modifications made available for spread to other hypertension improvement initiatives, including projects in various Quality Innovation Network-Quality Improvement Organizations and in the Agency for Healthcare Research and Quality funded project. ${ }^{[28]}$ More recently, the AMA has collaborated with the Care Coordination Institute. ${ }^{[29]}$ and the American Heart Association in Target: BP. ${ }^{[26]}$ The changes in the primary outcomes measured dur- ing the IHO: BP pilot may not reflect the potential impact of the program, but these efforts continue to offer valuable insights to the $I H O: B P$ project team on how best to engage physicians and care teams across the nation in the effort to improve blood pressure control and reduce the risks of heart attack and strokes.

\section{ACKNOWLEDGEMENTS}

The authors would like to thank the members of IHO: BP Collaboration and the AMA and Johns Hopkins who contributed to the design of the QI prototype. In addition, the authors would like to acknowledge the contributions of Annalynn Skipper from AMA Author Services for her review of the manuscript.

\section{CONFlicts OF INTEREST Disclosure}

The authors declare no conflicts of interest.

\section{DiSCLAIMER}

The findings and conclusions in this report are those of the authors and do not necessarily represent the official position of the AMA or Johns Hopkins.

\section{REFERENCES}

[1] Benjamin EJ, Blaha MJ, Chiuve SE, et al. Heart disease and stroke statistics-2017 update: a report from the American Heart Association. Circulation. 2017; 135(10): e146-e603. PMid:28122885. https://doi.org/10.1161/CIR.0000000000000485

[2] Wozniak G, Khan T, Gillespie C, et al. Hypertension Control Cascade: A Framework to Improve Hypertension Awareness, Treatment, and Control. The Journal of Clinical Hypertension. 2016; 18(3): 232-9. PMid:26337797. https://doi.org/10.1111/jch.12654

[3] Farley TA, Dalal MA, Mostashari F, et al. Deaths Preventable in the US by Improvements in Use of Clinical Preventive Services. American Journal of Preventive Medicine. 2010; 38(6): 600-609. PMid:20494236. https://doi.org/10.1016/j .amepre. 2010. 02.016

[4] Ritchey MD, Wall HK, Gillespie C, et al. Million Hearts: prevalence of leading cardiovascular disease risk factors-United States, 2005-2012. Morbidity and Mortality Weekly Report. 2014; 63(21): 462-467. PMid:24871251.

[5] Powers MA, Cuddihy RM, Bergenstal RM, et al. Improving blood pressure control in individuals with diabetes: a quality improvement collaborative. The Joint Commission Journal on Quality and Patient Safety. 2011; 37(3): 110-119. https://doi .org/10.1016/S155 3-7250 (11) 37013-4

[6] IHI. The Breakthrough Series: IHI's Collaborative Model for Achieving Breakthrough Improvement. Boston; 2003. Available from: Www. IHI.org

[7] Nembhard IM. Learning and improving in quality improvement collaboratives: which collaborative features do participants value most? Health services research. 2009; 44(2p1): 359-378.

[8] Nadeem E, Olin SS, Hill LC, et al. Understanding the components of quality improvement collaboratives: a systematic literature re- view. Milbank Quarterly. 2013; 91(2): 354-394. PMid:23758514. https://doi.org/10.1111/milq.12016

[9] Stevens DP, Bowen JL, Johnson JK, et al. A multi-institutional quality improvement initiative to transform education for chronic illness care in resident continuity practices. Journal of General Internal Medicine. 2010; 25(4): 574-580. PMid:20737232. https: //doi.org/10.1007/s11606-010-1392-z

[10] Wagner EH, Glasgow RE, Davis C, et al. Quality improvement in chronic illness care: a collaborative approach. The Joint Commission Journal on Quality and Patient Safety. 2001; 27(2): 63-80. https://doi.org/10.1016/S1070-3241(01) 27007-2

[11] Jager AJ, Choudhry SA, Marsteller JA, et al. Development and Initial Validation of a New Practice Context Assessment Tool for Ambulatory Practices Engaged in Quality Improvement. American Journal of Medical Quality. 2016; 32(4): 423-437. PMid:27469005. https://doi.org/10.1177/1062860616659132

[12] Boonyasai RT, Rakotz MK, Lubomski LH, et al. Measure accurately, Act rapidly, and Partner with patients: An intuitive and practical three-part framework to guide efforts to improve hypertension control. The Journal of Clinical Hypertension. 2017. PMid:28332303. https://doi.org/10.1111/jch.12995

[13] Rakotz M. Improving blood pressure control. American Medical Association StepsForward. Available from: https://www. stepsforward.org/modules/hypertensio n-blood-pressure-control. Published 2016. Accessed September 1, 2016.

[14] Daniel DM, Wagner EH, Coleman K, et al. Assessing Progress toward Becoming a Patient-Centered Medical Home: An Assessment Tool for Practice Transformation. Health services research. 2013; 48(6pt1): 1879-1897. PMid:24138593. https ://doi.org/10.1 $111 / 1475-6773.12111$ 
[15] Berenholtz SM, Needham DM, Lubomski LH, et al. Improving the quality of quality improvement projects. Joint Commission journal on quality and patient safety/Joint Commission Resources. 2010; 36(10): 468-473. https://doi.org/10.1016/S1553-7250(10 ) 36069-7

[16] Sawyer M, Weeks K, Goeschel CA, et al. Using evidence, rigorous measurement, and collaboration to eliminate central catheterassociated bloodstream infections. Critical Care Medicine. 2010; 38: S292-S298. PMid:20647786. https://doi.org/10.1097/CC M. 0b013e3181e6a165

[17] Goeschel CA, Holzmueller CG, Cosgrove SE, et al. Infection preventionist checklist to improve culture and reduce central lineassociated bloodstream infections. Jt Comm J Qual Patient Saf. 2010. https ://doi .org/10.1016/S1553-7250(10) 36085-5

[18] Hales BM, Pronovost PJ. The checklist-a tool for error management and performance improvement. Journal of Critical Care. 2006; 21(3): 231-235. PMid:16990087. https://doi.org/10.1016/j.jcrc .2006 .06 .002

[19] Parchman ML, Noel PH, Culler SD, et al. A randomized trial of practice facilitation to improve the delivery of chronic illness care in primary care: initial and sustained effects. Implementation Science. 2013; 8(1): 1. PMid:23965255. https://doi.org/10.1186/17 48-5908-8-93

[20] Baskerville NB, Liddy C, Hogg W. Systematic review and metaanalysis of practice facilitation within primary care settings. The Annals of Family Medicine. 2012; 10(1): 63-74. PMid:22230833. https://doi.org/10.1370/afm.1312

[21] Dowding D, Randell R, Gardner P, et al. Dashboards for improving patient care: review of the literature. International Journal of Medical Informatics. 2015; 84(2): 87-100. PMid:25453274. https://doi.org/10.1016/j.ijmedinf.2014.10.001
[22] Michtalik HJ, Carolan HT, Haut ER, et al. Use of provider level dashboards and pay for performance in venous thromboembolism prophylaxis. Journal of Hospital Medicine. 2015; 10(3): 172-178. PMid:25545690. https://doi.org/10.1002/jhm. 2303

[23] O'Neill E, Power A, Stevens N, et al. Effectiveness of podcasts as an adjunct learning strategy in teaching clinical microbiology among medical students. Journal of Hospital Infection. 2010; 75(1): 83-84 PMid:20227141. https://doi.org/10.1016/j.jhin.2009.11 .006

[24] Jalali A, Leddy J, Gauthier M, et al. Use of Podcasting as an Innovative Asynchronous E-Learning Tool for Students. Online Submission. 2011.

[25] Shantikumar S. From lecture theatre to portable media: students' perceptions of an enhanced podcast for revision. Medical Teacher 2009; 31(6): 535-538. PMid:18937140. https://doi.org/10.1 $080 / 01421590802365584$

[26] American Medical Association; American Heart Association. Target BP. Available from: https://targetbp. org. Published 2017. Accessed January 21, 2018.

[27] American Medical Association. Improving Health Outcomes. Available from: https://www.ama-assn.org/about/improving-h ealth-outcomes. Published 2016. Accessed January 21, 2018.

[28] American Medical Association. AMA Target: BP Program. Telligen Available from: https://telligenqinqio.com/ama-johns-h opkins-medicine-improving-health-outcomes-bp-progr am/. Published 2016. Accessed January 21, 2018.

[29] Hanlin RB, Asif IM, Wozniak G, et al. Measure Accurately, Act Rapidly, and Partner With Patients (MAP) improves hypertension control in medically underserved patients: Care Coordination Institute and American Medical Association Hypertension Control Project Pilot Study results. The Journal of Clinical Hypertension. 2018. PMid:29316149. https://doi.org/10.1111/jch.13141 